\title{
Influence of Socioeconomic Status on Anthropometric Measurements in Secondary School Children in Mumbai, India
}

\author{
Thakur Anuprita ${ }^{1}$, Yardi Sujata ${ }^{2}$, Arora Anu ${ }^{3}$ \\ ${ }^{1}$ Professor, School of Physiotherapy, D. Y. Patil University, Navi Mumbai, India \\ ${ }^{2}$ Ex-Director and Professor, School of Physiotherapy, D. Y. Patil University, Navi Mumbai, India \\ ${ }^{3}$ Associate Professor, School of Physiotherapy, D. Y. Patil University, Navi Mumbai, India
}

\begin{abstract}
Objective: This study assessed the influence of socioeconomic status (SES) on anthropometric measurements in secondary school children in Mumbai, India. Method: This was cross sectional study of 522 children between 12.5 to 15.5 years of age selected by convenient sampling from various schools in Mumbai and Navi Mumbai, Maharashtra, India. Anthropometric measurements like Body mass Index (BMI), Waist circumference (WC) and percent body fat was measured using standard techniques. SES was assessed using modified Kuppuswamy's socioeconomic status scale and school attended was used as proxy measure of socioeconomic status. Results and Conclusion: BMI, WC and percent body fat were observed to be more in children from upper SES group as compared to lower and middle SES groups. This study thus confirms the direct effect of socioeconomic status on anthropometric measurements in children.
\end{abstract}

Keywords: Anthropometric measurements, socioeconomic status, children

\section{Introduction}

Childhood obesity is a serious risk factor for the development of obesity in adulthood which increases the risk of cardiometabolic diseases in adulthood ${ }^{1,2}$. Childhood obesity is known to continue into adulthood ie .overweight children often become overweight adults ${ }^{3}$. Adolescence is one of the vulnerable periods of life for development of future obesity as this phase is associated with irregular and changed food habits, periods of inactivity during leisure combined with physiological change which promote increased fat deposition ${ }^{4}$. Therefore, it is imperative to monitor the body composition in children and adolescents in order to prevent the accumulation of excess body fat ${ }^{5}$.

Socio-economic status (SES) is considered as an important determinant of health and well-being because it influences people's attitudes, experiences, and exposure to several health risk factors ${ }^{6}$. It is known to be positively associated with better nutrition, housing, schooling, and recreation ${ }^{7}$. In children, SES would affect a wide array of health and socioemotional outcomes ${ }^{8}$. SES is known to be a confounding factor in development of childhood obesity ${ }^{9}$. This study thus aims to assess the influence of SES on anthropometric measurements in secondary school children in Mumbai, India.

\section{Literature Review}

Sobal et $\mathrm{al}^{10}$ reviewed 144 published studies to assess the relationship between SES and obesity. Primary finding included the observation of a strong direct relation between obesity and socioeconomic status for women, men, and children in developing societies. Another study supported this finding wherein it concluded that as one moved from high- to medium- to low-HDI (Human Development Index) countries, the proportion of positive associations increased and the proportion of negative associations decreased, for both men and women ${ }^{11}$.

\section{Methodology}

The permission to conduct the study was obtained from the University Ethics committee. This was an observational cross-sectional study which included 522 children (males288 , females- 234) in the age groups of 12.5 to 15.5 years selected through convenient sampling from various schools in Mumbai and Navi Mumbai. The age group between 12 to15 years was selected for this study as majority of the changes associated with adolescence take place and complete in this phase ${ }^{12}$. Children with known cardio-respiratory, musculoskeletal, neurological, metabolic and mental disorders and those participating in sports at a professional level were excluded from this study.

The modified Kuppuswamy Socioeconomic Status scale was used to assess the SES status of the children ${ }^{13}$. In addition, the type of school attended by the children was also taken as a proxy measure of SES. It is generally observed that in India, government/municipal schools cater to poorer sections of the population, whereas semi-private schools cater to lower middle and middle income groups. Children from upper middle and high income groups attend private schools ${ }^{14,15}$. Based on Kuppuswamy Socioeconomic Status scale and the school attended, the children in this study were classified in 3 SES groups: Upper SES (attending private school and SES score 26-29), Middle SES (attending semi-private school and SES score 11-25) and Lower SES (attending municipal school and SES score $<11$ ). 


\section{International Journal of Science and Research (IJSR) \\ ISSN (Online): 2319-7064 \\ Index Copernicus Value (2013): 6.14 | Impact Factor (2015): 6.391}

Body Mass Index (BMI) was calculated using the Quetelet Index ${ }^{16}$ by taking the child's weight (in kgs) and dividing by the height (in meters) squared. Children were classified into four categories (Underweight, Normal, Overweight or Obese) based on the BMI cut-off points given by the Indian Association of Pediatrics ${ }^{17}$ Waist circumference (in $\mathrm{cms}$ ) was measured at a site just above the iliac crest ${ }^{18}$. Subcutaneous fat was measured through skinfold calliper at tricpes and calf site $^{19}$. Percent body fat was computed through these measurements using the Slaughter- Lohman children formula $^{20 .}$

\section{Results}

The data was analyzed using SPSS version 20. One-way ANOVA was used to compare the means of the anthropometric measurements between the three SES groups and post hoc was done using Tukey-Bonferonni test. The level of significance was set at $<0.05$

Table 1a: SES wise distribution of boys on the basis of BMI

\begin{tabular}{|c|c|c|c|c|}
\hline & \multicolumn{4}{|c|}{ Boys } \\
\hline & Low SES & Middle SES & Upper SES & Total Males \\
\hline Under-weight & 21 & 17 & 8 & 46 \\
\hline Normal & 56 & 68 & 32 & 156 \\
\hline Over-weight & 10 & 18 & 23 & 51 \\
\hline Obese & 7 & 9 & 19 & 35 \\
\hline
\end{tabular}

Table 1b: SES wise distribution of girls on the basis of BMI

\begin{tabular}{|c|c|c|c|c|}
\hline & \multicolumn{4}{|c|}{ Girls } \\
\hline & Low SES & Middle SES & Upper SES & Total Males \\
\hline Under-weight & 16 & 14 & 5 & 35 \\
\hline Normal & 53 & 57 & 32 & 142 \\
\hline Over-weight & 10 & 17 & 13 & 40 \\
\hline Obese & 3 & 6 & 8 & 17 \\
\hline
\end{tabular}

Overall maximum children (54\% boys, $61 \%$ girls) from all socioeconomic groups showed BMI within normal ranges. More numbers of underweight boys and girls were observed in lower SES (46\%) as compared to middle (38\%) and upper SES groups $(16 \%)$. Overweight and obese children were seen more in the upper SES (44\%), followed by middle SES $(35 \%)$ and least in lower SES groups $(21 \%)$. Gender wise comparison showed more number of girls from low(22\%) and middle SES (40\%) were overweight and obese as compared to boys from the corresponding SES groups(19\% in low and $31 \%$ in middle).

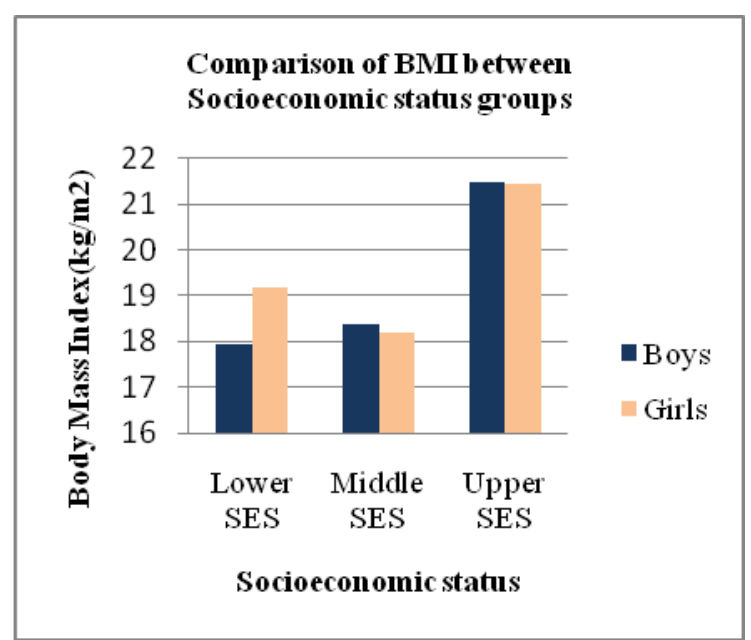

Figure 1: Comparison of BMI between SES groups

In both genders, the BMI was more in children from upper SES as compared to children from lower SES and middle SES and this difference was statistically significant $(<0.0001$ in boys and 0.007 in girls). Girls from lower SES were observed to have significantly higher BMI as compared to boys from the corresponding age group $(p=<0.0001)$.

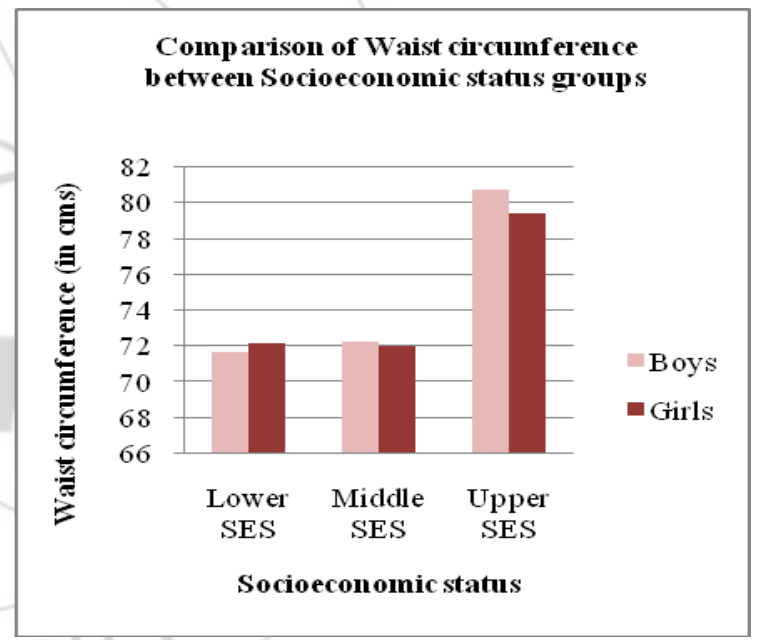

Figure 2: Comparison of Waist circumference between SES groups

Waist circumference was observed to be significantly more in both boys and girls from upper SES as compared to children from lower SES and middle SES and this difference was statistically significant ( $\mathrm{p}=<0.0001$ in both genders). No difference in waist circumference was observed between boys and girls of corresponding socioeconomic groups. 


\section{International Journal of Science and Research (IJSR) \\ ISSN (Online): 2319-7064}

Index Copernicus Value (2013): 6.14 | Impact Factor (2015): 6.391

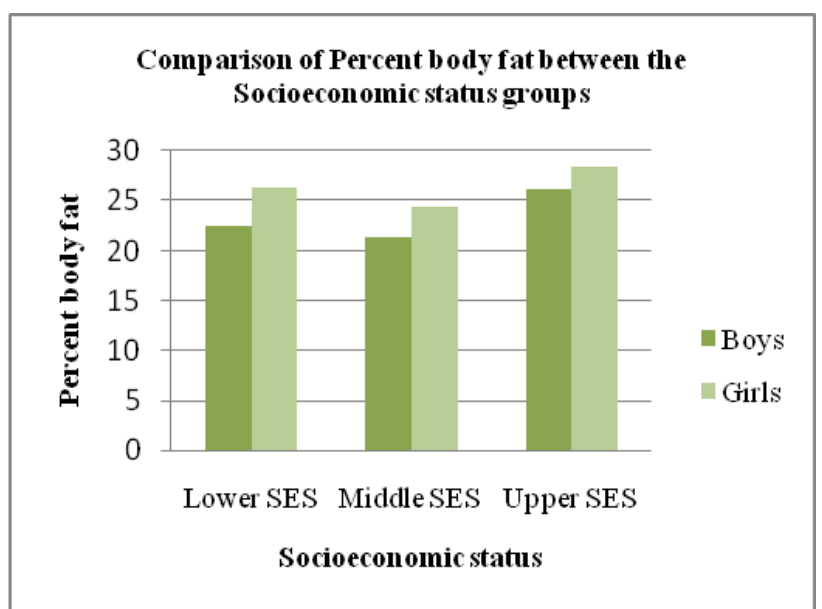

Figure 3: Comparison of Percent body fat between SES groups

Percent body fat was observed to be maximum in children from upper SES followed by children in lower SES and least in children from middle SES. The difference in percent body fat was statistically significant between lower and higher SES and middle and higher SES ( $\mathrm{p}=0.0003$ in boys and 0.0055 in girls). Girls from lower and middle SES showed significantly higher percent body fat as compared to boys from corresponding socioeconomic groups $(\mathrm{p}=0.001)$.

\section{Discussion}

Excess increase in body weight and body fat is the result of an energy imbalance due to energy intake having exceeded energy expenditure over a period of time ${ }^{21}$. Energy intake is in the form of food that is consumed by an individual while energy expenditure is through the physical activity or exercise performed by the person. The results in this study indicate that socioeconomic status does play a role in the development of overweightedness or obesity in adolescents.

Children from upper SES are more prone to increase in body weight and body fat as compared to those from middle and lower SES while more underweight children were seen in lower SES. Possible explanation for the different SESoverweight and obesity relationship is that SES may influence people's lifestyles such as diet, food consumption patterns, and access to public services such as health care and transportation and physical activity ${ }^{15}$.

Literature reports a replacement of the traditional diet of coarse grains and millets by refined wheat and rice as the staple cereal which has reduced the consumption of fibre content $^{22,23}$. A parallel increase in consumption of fats, oils, sugars, and western-style fast foods is also observed in the urban affluent ${ }^{22,23}$. Food cost might also play a significant role in determining eating patterns ${ }^{24}$. Increase in cost of fruits and vegetables lead to a decrease in their access to people of lower SES ${ }^{24,25}$. In addition, fast food culture is an emerging trend among the younger generation. The ready availability, taste, marketing strategies and peer pressure make them popular with children and adolescents ${ }^{26}$. This has led to more frequent consumption of meals at fast-food outlets, consumption of high calorie foods such as high fat, low-fiber foods, consumption of oversized portions at home and at restaurants and intake of sweetened beverages. These behaviours are more common in children from upper SES where the high calorie food is abundant, affordable, available, and easy to consume with minimal preparation as compared to children from middle and lower SES who probably have an occasional access to $\mathrm{it}^{25}$.

All these factors may be leading to an increase in BMI, waist circumference and percent fat observed in children from upper SES as against middle and lower SES.

In addition to food consumption, physical activity may also act as a confounding factor in altering the body composition in adolescents. A general decrease in physical activity levels was observed in children irrespective of the socioeconomic status $^{27}$. This may also contribute to decrease in energy expenditure thus leading to an excess increase in body fat. Children from middle SES probably fall in between the two extremes of abundance in upper SES and inadequacy in the lower SES and thus may be spared from the extreme influence of the various confounding factors.

\section{Conclusion}

From the results of this study, we can conclude that socioeconomic status did have a direct influence on the anthropometric measurements like BMI, waist circumference and percent body fat in school children.

\section{Conflict of Interest}

Nil

\section{References}

[1] Aronne L., Segal K. 'Adiposity and fat distribution outcome measures: assessment and clinical implications.' Obesity Research, 2002; 10 Suppl1:14S$21 \mathrm{~S}$

[2] Kaur S., Kapil U., Singh P. 'Pattern of chronic diseases amongst adolescent obese children in developing countries' Current Science 2005; 88(7): 1052-1056

[3] Serdula K, Ivery D, Coates J, Freedman S, Williamson F, Byers T. 'Do obese children become obese adults? A review of the literature.' Preventive Medicine 1993; 22:167-177.

[4] Braddon F., Rodgers B, Wadsworth M., Davies J. 'Onset of obesity in a 36 year birth cohort' British Medical Journal (Clinical Research edition) 1986; 293(6542): 299-303

[5] Goran M. 'Measurement issues related to studies of childhood obesity: Assessment of body composition, body fat distribution, physical activity and food intake' Pediatrics 1998;101(3 Pt 2):505-518

[6] K Park 'Park's Textbook of Preventive Medicine' $19^{\text {th }}$ edition, M/s Banarasidas Bhanot Publishers, India

[7] Adler N., Newman K. 'Socioeconomic disparities in health: Pathways and Policies' Health Affairs 2002; 21(2): $60-76$ 


\section{International Journal of Science and Research (IJSR) \\ ISSN (Online): 2319-7064}

Index Copernicus Value (2013): 6.14 | Impact Factor (2015): 6.391

[8] Bradley R., Corwyn R. 'Socioeconomic status and child development' Annual Review of Psychology 2002; 53: 371-399

[9] Jin Y., Jones Smith J. 'Associations between family income and children's physical fitness and obesity in California 2010-2012' Preventing Chronic Disease 2015; 12: 1-9

[10] Sobal J., Stunkard A. 'Socioeconomic status and obesity: a review of the literature'. Bulletin 1989; 105(2):260-75.

[11] Mc Laren L. 'Socioeconomic status and obesity' Epidemiologic Reviews 2007; 29: 29-48

[12] Kliegman R., Stanton B., St. Geme J. Schor N., Behrman R. 'Nelson Textbook of Pediatrics' Vol Part I-XVI, 2005, $19^{\text {th }}$ Edition, Pg 649-654, Elsevier Saunders, USA

[13] Anjan R., Pradeepa R., Das A., Mohan Deepa, Bhansali A., Joshi S., Joshi P. et al 'Physical activity and inactivity patterns in India - results from the ICMRINDIAB study (Phase-1) [ICMR-INDIAB-5]' International Journal of Behavioural Nutrition and Physical Activity 2014; 11: 26.

[14] Laxmaiah A., Nagalla B., Vijayaraghavan K., Nair M. 'Factors Affecting Prevalence of overweight among 12 to 17year old urban adolescents in Hyderabad, India' Obesity 2007; 15(6): 1384-1390

[15] Goyal R., Shah V., Saboo B., Phatak S., Shah N. et al 'Prevalence of overweight and obesity in Indian adolescent school going children: Its relationship with socioeconomic status and associated lifestyle factors' Journal of the Association of Physicians of India 2010; 58: $151-158$

[16] Stirbu M., Simalcsik A., Simalcsik R., Fedor C. 'Variability of the Quetelt Index (Body Mass Index) in 3-18 years old children and adolescent from two different ecological media' Anthropology, 2009:1; 37-43

[17] Khadilkar V., Yadav S., Agrawal K., et al 'Revised IAP growth charts for Height, weight and Body Mass Index for 5 to 18 year old Indian children' Indian Paediatrics, January 2015; 52: 47-55

[18]Conrnier Marc-Andre, Despres Jean-Pierre, Davis Nichola, Grossniklaus Daurice, Klein Samuel et al 'Assessing Adiposity- A scientific statement from American Heart Association' Circulation 2011; 124: 1996-2019

[19] Meredith M., Welk G. 'FITNESSGRAM test administration manual' $4^{\text {th }}$ edition, 2010, Human Kinetics, USA

[20] Slaughter M., Lohman T., Boileau R. et al 'Skinfold equations for estimation of body fatness in children and youth.' Human Biology 1988 Oct;60(5):709-23.

[21] Shetty P. 'Obesity in children in developing societies: Indicator of economic progress or a prelude to health disaster? Indian Pediatrics 1999; 36: 11-15

[22] Chaterjee P. 'India sees parallel rise in malnutrition and obesity' Feature in The Lancet 2002; 360:1948

[23] Vaz M., Yusuf S., Bharathi A., Kurpad A., Swaminathan $\mathrm{S}$. 'The nutrition transition in India' South African Journal of Clinical Nutrition 2005;18(2): 198-201

[24] Popkin B., Duffey K., Gordon-Larse P. 'Environmental influences on food choice, physical activity and energy balance' Physiology \& Behavior 2005; 86: 603 - 613
[25] Manu R., Krishna Kumar 'Obesity in children and adolescents' Indian Journal of Medical Research 2010; 132(5): 598-607

[26] Kaushik J., Narang M., Parakh A. 'Fast food consumption in children' Indian Pediatrics 2011; 48:97101

[27] Currie C., Roberts C., Morgan A., Smith R., Settertobulte W., Samdal O. et al, editors 'Young people's health in context: Health behaviour in schoolaged children (HSBC) study: International report from the 2001/2002 survey' Geneva: World Health Organization; 2004.

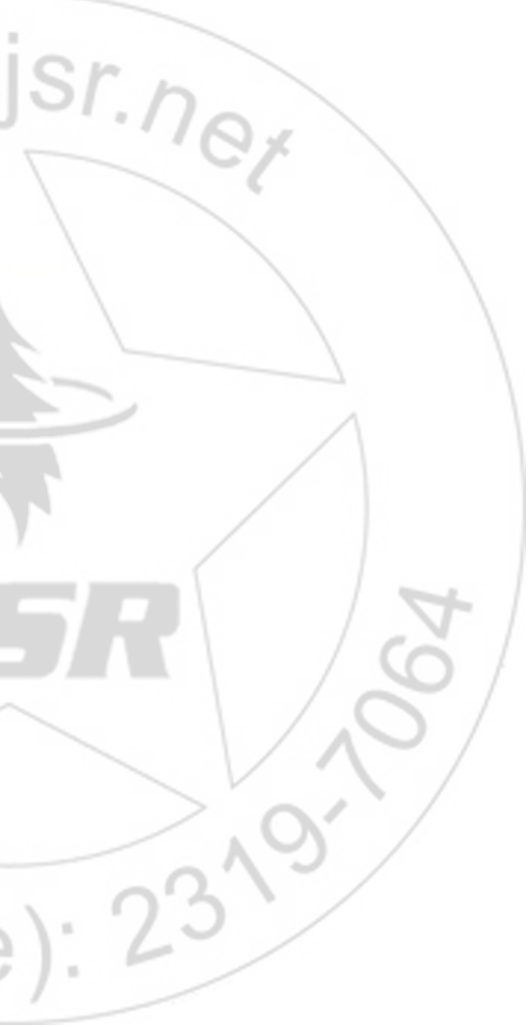

